\title{
Towards a new generation of fibre-optic chemical sensors based on spider silk threads
}

\author{
Kenny Hey Tow ${ }^{a}$, Desmond M. Chow ${ }^{a}$, Fritz Vollrath ${ }^{b}$, Isabelle Dicaire ${ }^{c, e}$, Tom Gheysens ${ }^{d, e}$ \\ and Luc Thévenaz ${ }^{a}$ \\ ${ }^{a}$ EPFL Swiss Federal Institute of Technology, Group for Fibre Optics, SCI-STI-LT, Station 11, \\ CH-1015 Lausanne, Switzerland; \\ ${ }^{b}$ University of Oxford, Department of Zoology, Oxford OX1 3PS, UK; \\ ${ }^{c}$ CCTT Optech, 1111 Lapierre St., Montreal QC, H8N 2J4, Canada; \\ ${ }^{d}$ Polymer Chemistry \& Biomaterials Research Group, Ghent University, Krijgslaan 281, \\ S4-Bis, B-9000 Ghent, Belgium; \\ ${ }^{e}$ Advanced Concepts Team, European Space Agency (ESA), Noordwijk, The Netherlands.
}

\begin{abstract}
A spider uses up to seven different types of silk, all having specific functions, to build its web. For scientists, native silk - directly extracted from spiders - is a tough, biodegradable and biocompatible thread used mainly for tissue engineering and textile applications. Blessed with outstanding optical properties, this protein strand can also be used as an optical fibre and is, moreover, intrinsically sensitive to chemical compounds. In this communication, a pioneering proof-of-concept experiment using spider silk, in its pristine condition, as a new type of fibre-optic relative humidity sensor will be demonstrated and its potential for future applications discussed.
\end{abstract}

Keywords: Chemical sensing, optical fibre sensor, spider silk, relative humidity sensor

\section{INTRODUCTION}

Nowadays, silica fibres are extensively used to monitor different parameters such as temperature and strain in optical fibre sensing systems. They are even used in fibre-optic chemical sensors (FOCS) although silica is, by essence, chemically inert thus making these fibres an improper material for detecting chemical species. In fact, the silica optical fibre is reduced to, merely, the role of a convenient photon conveyor to bring light to and from a chemically-sensitive coating and is not exploited as the sensing element in FOCS. Their sensing mechanism is either indirect - the reaction between the analyte and a doped fibre-tip end modifies the environmental quantities for which silica is sensitive - or part of the light must be made to propagate out of the silica fibre, for instance by evanescent field, and interact with a chemically sensitive coating placed on the fibre. In the first case, the light interaction is confined to a restrictive sensing area (fibre tip) while for the latter the waveguiding properties of the silica fibre is clearly a penalty, making these systems not very efficient

Recent improvement in the transparency and the fabrication process of Polymer Optical Fibres (POFs), have encouraged their use for optical fibre sensing. ${ }^{1}$ Their inherent properties - high elastic strain limits, fracture toughness, flexibility, and potential negative thermo-optic coefficients - offer significant advantages for temperature and strain sensing-based applications, but no real added value or, at the very least, only incremental improvement for chemical sensing since these fibres are also not intrinsically sensitive and need to be activated by a cladding material or coated with a chemical sensitive material when integrated in fibre-based sensors to detect chemical species; $;^{2,3}$ the only advantage of using POFs instead of their silica counterparts being the fact that their surface properties can be more easily manipulated using simple and wide-ranging chemistry techniques to achieve the desired sensing application.

Further author information: send correspondence to Kenny Hey Tow (kenny.heytow@epfl.ch)

25th International Conference on Optical Fiber Sensors, edited by Youngjoo Chung, Wei Jin,

Byoungho Lee, John Canning, Kentaro Nakamura, Libo Yuan, Proc. of SPIE Vol. 10323,

103231E - C 2017 SPIE · CCC code: 0277-786X/17/\$18 - doi: 10.1117/12.2264438

Proc. of SPIE Vol. 10323 103231E-1 
A radically novel approach to drastically improve the performance of FOCS is to use an optical fibre made from a material, which directly reacts with targeted chemical species in its pristine condition. Under the influence of modifying agents, the fibre's properties will be altered in its entire volume. This change will, in turn, subtly alter the parameters (intensity, phase, spectrum, polarisation, etc.) of light propagating along the sensing fibre. By collecting and analysing the transmitted light, the presence of the modifying agent can be detected using a very simple transmission setup as shown in figure 1. In this configuration, the optical fibre is both used as the light conveyor and the sensing element along its whole length. Therefore, the benefits of optical fibre sensing is entirely exploited since the guided light keeps fully confined in the fibre and the effect is accumulated along the fibre, thereby upscaling its sensitivity. Natural fibres can prove to be an inspir-

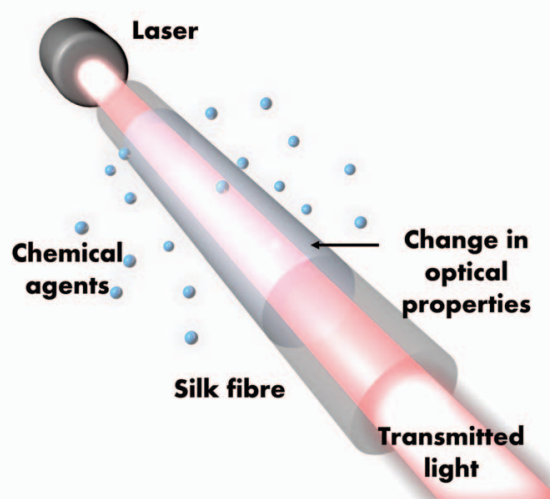

Figure 1: Simple transmission setup proposed for our spider silk-based FOCS. ing option since they are capable of interacting with the surrounding environment. For instance, hydrophilic materials such as cellulose fibrils in wheat awns, horse hair, wool, etc. all swell during bulk water uptake. These specific conformational change will lead to a signature change in material properties of the fibre bulk material, which can be measured by the light propagating along these natural threads.

\section{SPIDER SILK THREADS AS CHEMICALLY-SENSITIVE OPTICAL FIBRES}

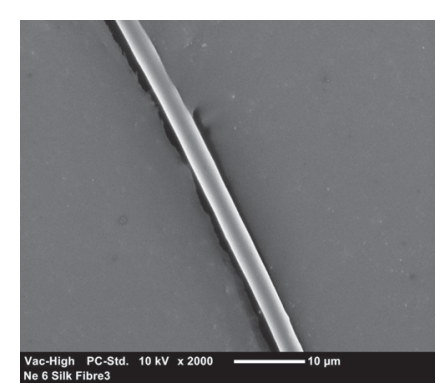

(a)

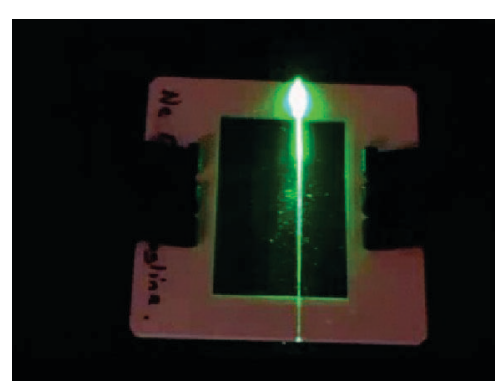

(b)

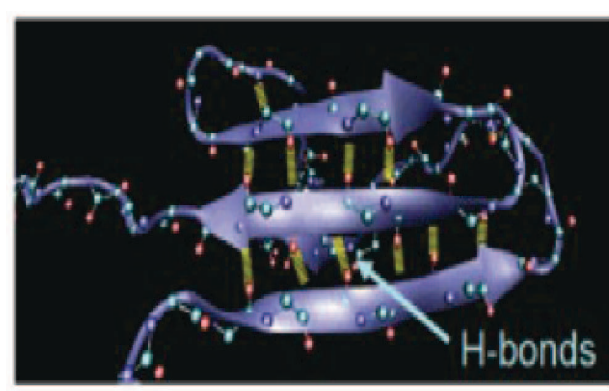

(c)

Figure 2: (a) Scanning electron microscope (SEM) image of a dragline silk, collected by the Oxford Silk Group. (b) A sample of dragline silk used as an optical fibre. (c) Close-up of spider silk protein with hydrogen bonds (yellow) holding the beta-sheet assembly together. ${ }^{4}$

Silk is, in particular, a very appealing biomaterial to implement our proposed sensing method. When reeled under controlled conditions, dragline silk - directly extracted from the major ampullate glands of a spider - can be uniformly spun into a silk thread with smooth surface and equal circular diameter (figure 2(a)). In its pristine condition, dragline silk transmits light (figure $2(\mathrm{~b})$ ), with propagation losses of the order of a few $\mathrm{dB} / \mathrm{cm}$ within a measured transparency window from the visible up to $\lambda \approx 1400 \mathrm{~nm}$ and under high birefringence. ${ }^{5,6}$

Dragline silk is also known to be reactive to different classes of chemical species (acids, bases, etc.). When these modifying agents are present in the close vicinity of the silk, they affect the birefringence of the silk fibre material. ${ }^{7}$ For instance, exposure to humidity results in infiltration of water molecules into the silk fibre. The water molecules bind themselves to the random coils within the amorphous region, disrupting the relatively weak hydrogen bonding holding together the protein threads (figure 2(c)). This relaxes the silk, causing it to contract. ${ }^{8}$

\section{RELATIVE HUMIDITY FIBRE-OPTIC SENSOR BASED ON SPIDER SILK}

Dragline spider silk sample, directly reeled at a controlled speed of $5 \mathrm{~mm} / \mathrm{s}$ from a female Nephilia edulis spider, was used as a chemically-active optical fibre to measure ambient relative humidity ( $\mathrm{RH}$ ) level. A $2.5 \mathrm{~cm}-\mathrm{long}$ 
sample, kept at the same reeling tension, was placed on a holder and clamped at the two ends parts. Since the fibre is refrained from shrinking along its length, the latter swells when exposed to water molecules, which results in a change in the silk fibre's geometry on its whole volume.

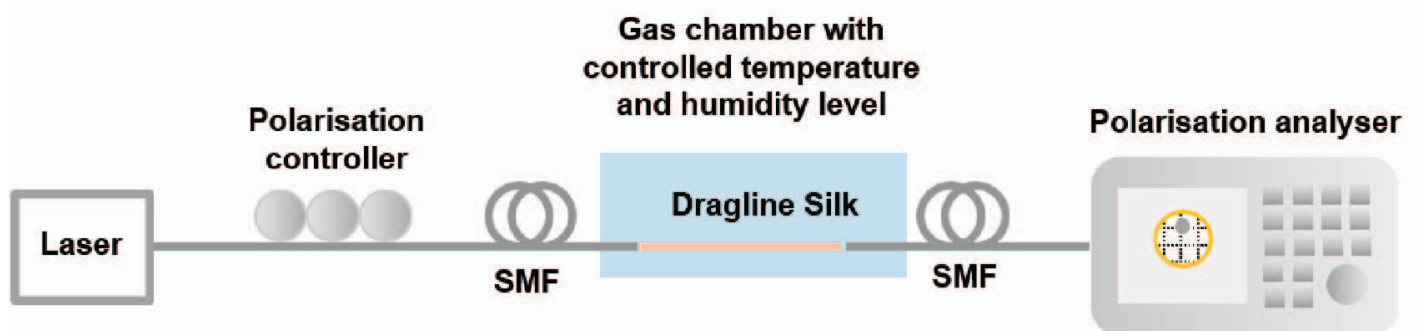

Figure 3: Polarimetric setup used in our experiment. SMF: Single mode fibre

A polarimetric setup, schematised in figure 3, was used to validate our proposed concept. Linearly polarised light, in the O-band region, was launched into the silk fibre by end-fire injection and the SOP of the collected light was measured with a polarisation analyser. The silk thread was placed inside an hermitically sealed gas chamber at constant pressure (MKS Baratron pressure sensor) and temperature (PT1000 temperature sensor) to ensure that these varying external ambient conditions did not affect our result. In particular, temperature is known to highly affect the mechanical properties of $\operatorname{silk}^{8}$ at high temperatures, much less though in the room temperature range where changes in humidity levels predominate over temperature changes in the silk's response.

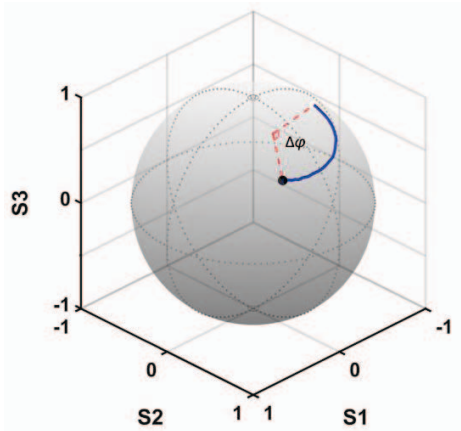

(a)

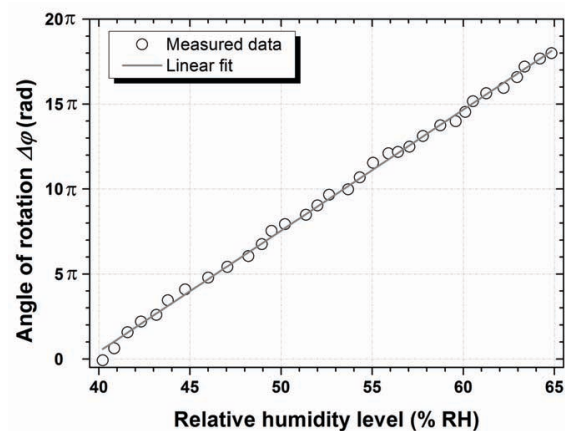

(b)

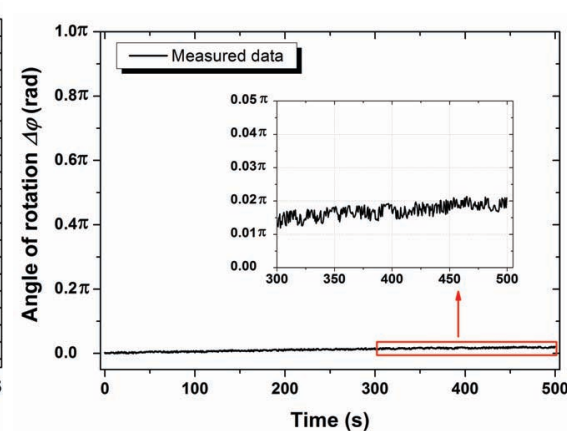

(c)

Figure 4: (a) Evolution of the SOP of the output light, represented on a Poincare sphere, when the RH level was slightly changed inside the gas chamber. (White dot: initial SOP; black dot: final SOP; blue line: SOP transformation path.). Measured phase shift when the relative humidity level was (b) increased from 40 to $65 \%$ and (c) kept constant in the gas chamber (Inset: zoom between $t=300 \mathrm{~s}$ and $\mathrm{t}=500 \mathrm{~s}$ ).

The sensor was evaluated over a relative humidity level range from 40 to $65 \%$ to avoid working in the supercontraction regime, ${ }^{9}$ which occurs at high humidty level of $70 \%$ and above. As soon as the first few drops of water vapour were introduced inside the chamber, the silk thread immediately reacted and a substantial change in the SOP of the output signal could be observed, translated in a rotation of $\Delta \varphi$ on the Poincare sphere as shown in figure 4(a). The RH level was gradually increased to $65 \%$ and the corresponding change in $\Delta \varphi$ was measured. The results are plotted in figure 4(b): a linear response with a sharp slope of $\sim 0.71 \pi \mathrm{rad} / \% \mathrm{RH}$, which reflects the high sensitivity over this range of the sensor. When the RH level in the chamber was decreased from 55 to $40 \%$, a sensitivity of $\sim-0.72 \pi \mathrm{rad} / \% \mathrm{RH}$ was obtained, which corresponds to almost the same value measured for increasing humidity. On the other hand, $\Delta \varphi$ barely changed when we performed the experiment at fixed RH level; only a negligible drift of $0.02 \pi$ rad was recorded during a measurement time of $500 \mathrm{~s}$ (figure 4(c)).

\section{DISCUSSION}

The proof-of-concept of using a protein-based thread as a sensitive optical fibre for chemical sensing has been demonstrated. In this communication, a spider dragline silk was used as a fibre-optic sensor to measure ambient 
relative humidity content. Having a complex molecular structure, silk is also reactive to many classes of chemical compounds in its pristine condition and can, therefore, be used in a similar way as described in section 3 for sensing other target agents in trace amounts such as ammonia, ethanol, etc. Silk threads, therefore, represent a much better alternative for fibre-optic chemical sensing than conventional glass fibres since the latter usually require coatings, even to be used as relative humidity sensors. ${ }^{10}$ Moreover, they are spun at ambient conditions and offer the possibility of the incorporation of dyes and specific colorimetric reagents into the bulk material, which would otherwise be denatured at the very high drawing temperatures of $\sim 2000{ }^{\circ} \mathrm{C}$ for silica fibres.

All these qualities make these protein threads very promising for future applications. However, along this "silk road" there are still many barriers to overcome for us to fully exploit this highly promising material for optical fibre sensing. Even though they can be used as localised sensors despite their relatively high transmission losses, it is difficult to conceive a distributed chemical fibre sensing system based on these natural fibres and while they are excellent fibres for proof of concept and for fundamental research, native spider threads are unlikely to be accepted by industry for upscaling of the concept into commercial devices. Hopefully, the recent progress achieved in the understanding of the silk proteins and the drawing of artificial silk ${ }^{11}$ will allow the production of tailor-made low-loss synthetic optical threads. Ideally, this would be combined with an ability to fabricate functionalised fibres for selective sensing of specific chemical compounds.

\section{ACKNOWLEDGMENTS}

The authors would like to thank the European Space Agency for initiating this research work through the Ariadna study no $14-6401$ (The Silky Way).

\section{REFERENCES}

[1] Koike, Y. and Koike, K., "Progress in low-loss and high-bandwidth plastic optical fibers," Journal of Polymer Science Part B: Polymer Physics 49(1), 2-17 (2011).

[2] Goicoechea, J., Zamarreño, C. R., Matias, I., and Arregui, F., "Optical fiber ph sensors based on layer-bylayer electrostatic self-assembled neutral red," Sensors and Actuators B: Chemical 132(1), 305-311 (2008).

[3] Angelini, E., Grassini, S., Mombello, D., Neri, A., Parvis, M., and Perrone, G., "Plasma modified pof sensors for in situ environmental monitoring of museum indoor environments," Applied Physics A 100(3), 975-980 (2010).

[4] Keten, S. and Buehler, M. J., "Geometric confinement governs the rupture strength of h-bond assemblies at a critical length scale," Nano Letters 8(2), 743-748 (2008).

[5] Huby, N., Vié, V., Renault, A., Beaufils, S., Lefèvre, T., Paquet-Mercier, F., Pézolet, M., and Bêche, B., "Native spider silk as a biological optical fiber," Applied Physics Letters 102(12), 123702 (2013).

[6] Chow, D. M., Tow, K. H., Vollrath, F., Dicaire, I., Gheysens, T., and Thévenaz, L., "Shedding light on the optical properties of spider silk fiber," Proc. IEEE Photonics Conference 2015, 333-334 (2015).

[7] Tow, K. H., Chow, D. M., Vollrath, F., Dicaire, I., Gheysens, T., and Thévenaz, L., "Spider silk: a novel optical fibre for biochemical sensing," Proc. SPIE 9634, 96347D-96347D-4 (2015).

[8] Guan, J., Vollrath, F., and Porter, D., "Two mechanisms for supercontraction in nephila spider dragline silk," Biomacromolecules 12(11), 4030-4035 (2011).

[9] Work, R. W., "Dimensions, birefringences, and force-elongation behavior of major and minor ampullate silk fibers from orb-web-spinning spiders?the effects of wetting on these properties," Textile Research Journal 47(10), 650-662 (1977).

[10] Yeo, T., Sun, T., and Grattan, K., "Fibre-optic sensor technologies for humidity and moisture measurement," Sensors and Actuators A: Physical 144(2), 280-295 (2008).

[11] Rising, A. and Johansson, J., "Toward spinning artificial spider silk," Nature chemical biology 11(5), 309-315 (2015). 